\title{
Thin scintillators for ultrafast hard X-ray imaging
}

\author{
Zhehui Wang ${ }^{* a}$, Cris W. Barnes ${ }^{\mathrm{a}}$, Jon S. Kapustinsky ${ }^{\mathrm{a}}$, Chris L. Morris ${ }^{\mathrm{a}}$, \\ Ron O. Nelson ${ }^{\mathrm{a}}$, Fan Yang ${ }^{\mathrm{b}}$, Liyuan Zhang ${ }^{\mathrm{b}}$, Ren-Yuan Zhu ${ }^{\mathrm{b}}$ \\ ${ }^{a}$ Los Alamos National Laboratory, Los Alamos, NM 87545, U.S.A.; \\ ${ }^{\mathrm{b}}$ California Institute of Technology, Pasadena, CA 91125, U.S.A.
}

\begin{abstract}
A multilayer thin-scintillator concept is described for ultrafast imaging. The individual layer thickness is determined by the spatial resolution and light attenuation length, the number of layers is determined by the overall efficiency. By coating the scintillators with a high quantum-efficiency photocathode, single X-ray photon detection can be achieved using fast scintillators with low light yield. The fast, efficient sensors, when combined with MCP and novel nanostructed electron amplification schemes, is a possible way towards $\mathrm{GHz}$ hard X-ray cameras for a few frames of images.
\end{abstract}

Keywords: GHz X-ray imaging, MCP-PMT detectors, fast scintillators, detection efficiency.

\section{INTRODUCTION}

Compared with direct X-ray detections using semiconductors such as silicon, indirect detections using scintillators for ultrafast hard X-ray imaging ( 100 ps interframe time) have some known advantages and disadvantages. The main advantage is the large stopping power due to their large effective atomic numbers or mass density in many scintillators. One millimeter thick scintillators materials $\left(\mathrm{BaF}_{2}, \mathrm{LSO}\right)$ are sufficient to stop most $\mathrm{X}$ rays, resulting in high detection efficiency for $42 \mathrm{keV}$ photons [1]. The speed of light in scintillators is reduced from the speed of light in vacuum by the refractive index, which is typically in the range of 1-2. Therefore scintillator light is about 1000 times the saturated electron drift velocity in semiconductors, allowing sensor response time on the order of $10 \mathrm{ps}$. On the other hand, emission of scintillator light from an excited state of atom can take much longer than 10 ps, depending on the atomic properties as well as ambient conditions. Ultrafast photodetectors have to be used to convert the scintillator light to electrical charge towards the end; therefore, the speed and inter-frame time of a scintillator camera are typically not limited by light propagation in scintillators, but rather by the intrinsic atomic properties of the scintillator, as well the performance of photodetectors such as the quantum efficiency of photoelectric conversion and recovery time of the photodetectors for multi-frame imaging.

The spatial resolution of a scintillator camera is also limited by the scintillator thickness as illustrated by $\mathrm{BaF}_{2}$ here. The refractive index of $\mathrm{BaF}_{2}$ is 1.54 at $220 \mathrm{~nm}$. For an air-to-scintillator interface, the total internal reflection angle $\left(\theta_{c}\right)$ is asin $(1 / 1.54)=0.71$ for a flat interface. At incident angles less than $\theta_{c}$, both transmission and reflection may occur at the interface and the transmittance depends on both the polarization and incident-angle, as described by the Fresnel equations. The spatial resolution is limited to $2 \mathrm{~d} \sin \theta_{\mathrm{c}}$ for a thickness $\mathrm{d}$, assuming the light interacts with the interface only once $(\sim 95 \%$ of the light for incidental angles less than the Brewster's angle of atan $(1 / 1.54)=0.57)$. For $100-\mu \mathrm{m}$ spatial resolution, d cannot exceed $71 \mu \mathrm{m}$. Micron and submicron resolutions using thin film

\footnotetext{
*Email: zwang@lanl.gov; phone: (505) 665535
} 
scintillators have been reported for YAG:Ce [2], $\mathrm{NaI}(\mathrm{Tl})$ [3], LuAG:Ce, LuAG:Pr and a few others [4], when high-speed multi-frame imaging were not required. In addition, the characteristic decay time, combined with the light output, of a scintillator also limits the time response of a scintillator camera. Some of the fastest scintillators are $\mathrm{BaF}_{2}\left(0.65 \mathrm{~ns}\right.$ for $220 \mathrm{~nm}, 1.8$ photons/ $\left.\mathrm{keV} \mathrm{e}^{-}\right)$and $\mathrm{CsF}(2 \mathrm{~ns}$ with a peak emission wavelength of $390 \mathrm{~nm}$, light yield is 2.5 photons/ $\mathrm{keV} \mathrm{e}^{-}$). Plastic scintillators such as EJ-212 have a fast decay time about $2.4 \mathrm{~ns}$, light yield 8.4 photons $/ \mathrm{keV} \mathrm{e}^{-}$, but relatively low density around $1.0 \mathrm{~g} / \mathrm{cm}^{3}$. The relative low- $\mathrm{Z}$ in plastic scintillators implies that Compton scattering is significant when used to detect hard $\mathrm{X}$-rays at $40 \mathrm{keV}$ and above energies.

Here we describe a concept to coat thin scintillators with a thin photocathode layer, and to remove additional materials between the scintillators and photoelectron production and therefore maximizes the light coupling from the scintillator to signals, as shown in Figure 1. This approach is important since the amount of light emitted per X-ray photon in fast scintillators are typically quite small. We then discuss other components of a fast hard X-ray camera system, and a possible means for material/structure down-selection.

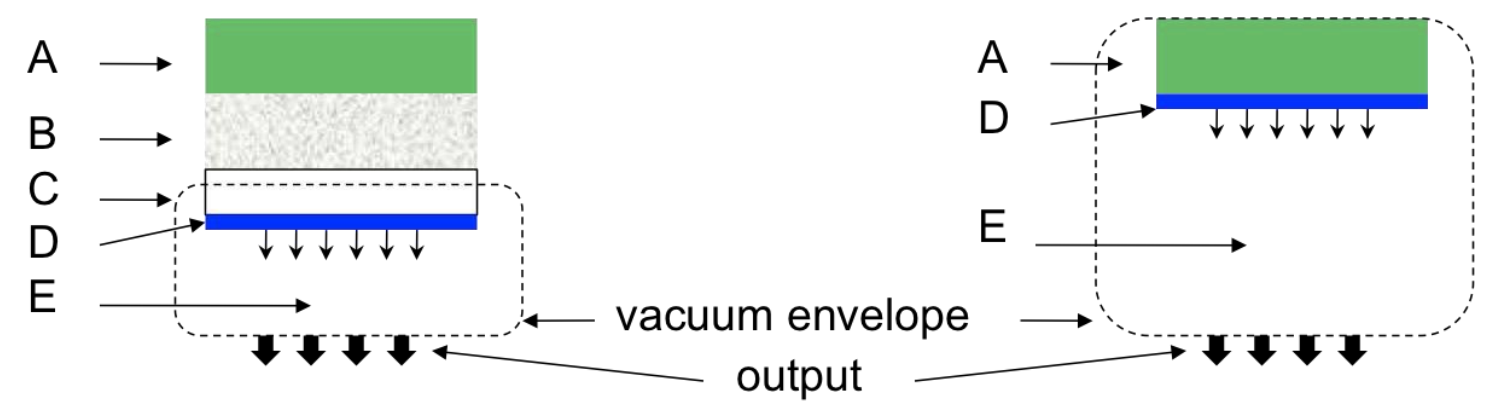

Figure 1: (left) existing approach to scintillator-based imaging. (Right) The proposed approach to scintillator-based imaging for low light-yield fast scintillators.

\section{FEASIBILITY FOR MULTI-FRAME ULTRAFAST IMAGING}

Single-frame ultrafast imaging does not have to use an 'ultrafast' camera. In a pump-probe experiment (PPE) when a short-pulse probe laser is delayed from a short-pulse pump laser, a slow camera that integrates all the signals from the experiment is sufficient to examine ultrafast evolution of material properties by, for example, repetitive PPE experiments with adjusted time delays between the pump and the probe. Charge-coupled devices (CCDs) are popular camera choices because of their low noise and gain uniformity (the same amplification is applied to all pixels during readout). A slow CCD camera used in a PPE collects (integrates) background most of the time, the fluctuations of which need to be much less than the signals from PPE. In other experiments, when either the pump or probe can not be timed precisely, or when multiple frames of images are needed during a single PPE, an ultrafast multi-frame camera would be needed. In a shock-driven experiment, for example, the timing of shock can be quite coarse $(\sim 1 \mathrm{~ns}$ or longer) compared with that of an ultrafast laser pulse $(<1 \mathrm{ps})$. An example of the latter is that when the PPE is too expensive or difficult to reproduce for many times, the information about the dynamic evolution of materials has to be gathered in a single experiment. $\mathrm{X}$-ray free electron lasers (XFEL) make both classes of experiments possible if an ultrafast camera is available. Under these scenarios, the precise timing between the camera and the XFEL can be used to 
record the ultrafast event, even when the precise timing of the shock is unknown. One of the remaining challenges is ultrafast multi-frame hard X-ray imaging camera technology.

In both the direct and the indirect schemes of hard X-ray imaging, photoelectron generation precedes a chain of signal generation, propagation, and amplification events until the signal is recorded. Detection of individual $X$ rays is independent of each other, even when the $X$ rays are coherent and when constructive and destructive interferences are expected at the detector location. An X-ray produces a photoelectron in the sensor through photoelectric absorption or Compton scattering. Coherent scattering of $\mathrm{X}$ ray in the sensor, as well as the transmitted or unscattered X-ray loss, does not give rise to any detectable signals and reduces detection efficiency. After a photoelectron is produced by an $\mathrm{X}$ ray, which has a kinetic energy $\mathrm{E}_{0}=\mathrm{h} v-\mathrm{I}$ in the case of photoabsorption, or $\mathrm{E}_{0} / \mathrm{h} v$ $=1-1 /\left[1+\mathrm{h} v / \mathrm{m}_{\mathrm{e}} \mathrm{c}^{2}(1-\cos \theta)\right]$ for Compton scattering. Here I is the binding energy of the photoelectron. We ignore I in Compton scattering, since Compton photoelectrons mostly come from the outer shells. The hole created by photoelectric absorption can lead to additional photoelectrons that will further contribute to light production. The process of X-ray absorption, photoelectron production can be simulated using various MC codes such as GEANT4.

The spatial resolution of the detector, as determined by the continuous slowing-down approximation (CSDA) of photoeletrons or the secondary particles such as Auger electrons, decreases with X-ray energy. For X-ray energies less than $100 \mathrm{keV}$, the CSDA ranges of the corresponding photoelectrons, as obtained from the NIST ESTAR database ${ }^{\dagger}$, are in the range of a few to $100 \mu \mathrm{m}$, shown in Figure 2. The uncertainties of the error are $2 \%$ to $3 \%$ in low- $\mathrm{Z}$ materials) and $5 \%$ to $10 \%$ in high- $\mathrm{Z}$ materials between $10 \mathrm{keV}$ and $100 \mathrm{keV}$.

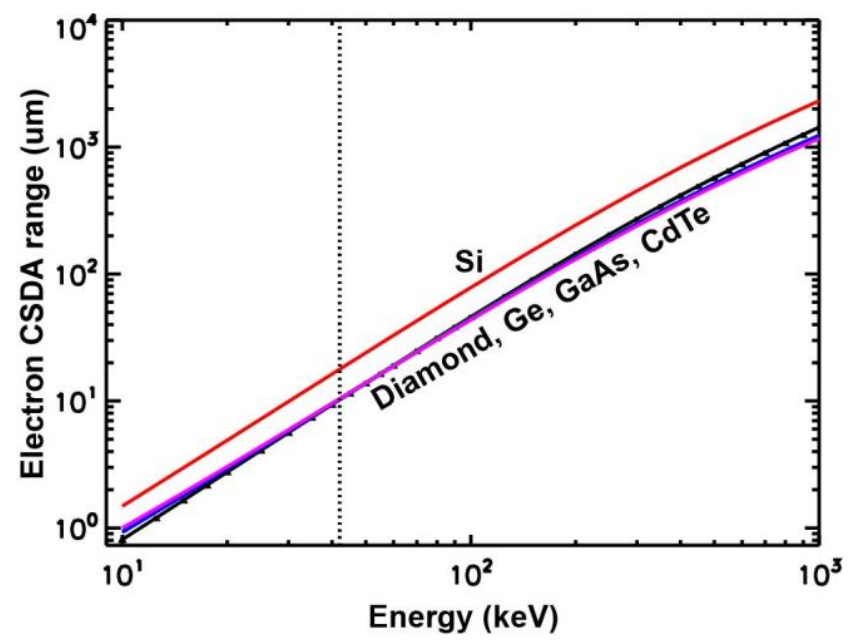

Figure 2. The CSDA range of electrons in various detector materials as a function of electron energy.

Visible/UV light is emitted along the paths of photoelectrons in scintillators. It is commonly assumed that the light is emitted isotropically into the $4 \pi$ solid angle from each emission location. Selfabsorption and coherent (Rayleigh) scattering cause further scintillation light loss within the bulk and

\footnotetext{
${ }^{\dagger}$ http://www.nist.gov/pml/data/star/
} 
degrade spatial resolution. Each loss mechanism is describable by an attenuation length. By coating a scintillator with a photocathode as in Fig. 1, the light transport loss is reduced at the interface since the light paths are minimized [5]. Visible/UV Light transport until a secondary photoelectron is produced. Multiplication of the secondary photoelectrons. Collection of the secondary photoelectrons, typically by capacitors, as stored signals. The multi-frame ultrafast cameras will also require that several storage capacitors be used, one for each frame of image. Therefore ultrafast X-ray sensing and ultrafast data recording are required at minimum for multi-frame ultrafast imaging. Ultrafast data transmission from the storage capacitors to a permanent data depository is not required since onboard data storage allows data download at a later time, assuming that the storage capacitors can hold the charge for a sufficiently long time.

\subsection{Thin vs bulk (thick) scintillators}

The UV grade $\mathrm{BaF}_{2}$ crystals by Korth Kristalle $\mathrm{GMBH}$, Fairfield Crystal technologies, and other vendors have an UV absorption edge near $137 \mathrm{~nm}$. The transmission at $220 \mathrm{~nm}$ approaches $90 \%$ for 5-mm thick crystals. $\mathrm{ZnO}$, a fast scintillator for near bandgap emissions, is traditionally discounted for applications when a large volume of detector mass is required because a.) They come in powder form and it is difficult to make large bulk crystals; and b.) The scintillator light self-absorption is quite strong. On the other hand, these scintillators are attractive to ultrafast hard X-ray imaging because of their light decay time can be comparable to or better than $\mathrm{BaF}_{2}$. Like synthetic diamond, wideband gap semiconductors are also radiation hard and are being pursued for power electronics, clean energies applications and radiation hard detectors in high-intensity frontiers. There is essentially no other inorgnic crystalline scintillator with a decay time constant less than $1 \mathrm{~ns}$. The potential of doped $\mathrm{ZnO}$ for fast detectors were reported previously [6] and the results are consistent with a few other studies. For example, the $380 \mathrm{~nm}$ free exciton transition in a $\mathrm{ZnO}$ single crystal from Eagle Picher shows a radiative lifetime of $440 \mathrm{ps}$ at $300 \mathrm{~K}$ and $290 \mathrm{ps}$ at $85 \mathrm{~K}$, and less than $100 \mathrm{ps}$ lifetime at lower temperature [7]. Various $\mathrm{ZnO}$ single crystals grown by Cermet, Inc. with different doping levels exhibit pulse rise times less than 100 ps and fall times shorter than 1 ns and light output comparable to plastic scintillator [8].

About $1 \mathrm{~mm}$ thick $\mathrm{ZnO}$ is required for high efficiency detection of $42 \mathrm{keV} \mathrm{X}$ rays, which is still too thick because of self-absorption. By using a multilayer detector architecture as described below, the material thickness of individual layers can be reduced to about $100 \mu \mathrm{m}$ or less, multiple layers together can be used to obtain high efficiency. Meanwhile, by using thin scintillators, a spatial resolution $\sim 100$ micron or less may also be possible, comparable to that in charge-based hard X-ray imaging. In addition to reducing the thickness of the sensor, another idea to reduce self-absorption is to shift the band-gap edge through high-level doping. Quantum size confinement effect can also widen the bandgap, but it only occurs in nano-meter scale objects. In InSb, Burstein-Moss effect has been demonstrated when the absorption band edge is shifted through n-doping. A quantum cascade laser has been built using the highly doped InSb at $3 \mu \mathrm{m}$, an otherwise strongly absorbed wavelength in InSb. In $\mathrm{ZnO}$, the band edge shift has been reported to be proportional to $\mathrm{n}^{2 / 3}$ for a carrier concentration $6 \times 10^{18}<\mathrm{n}<3 \times 10^{19} \mathrm{~cm}^{-3}$ with a shift up to $\sim 0.1 \mathrm{eV}$ [9]. Larger shift in energy through Al doping was also reported [10]. Ga doping concentration as high as $10^{21} \mathrm{~cm}^{-3}$ (2 at\%) was reported [11]. The band edge is also affected by the substrate [12]. 
We discuss the possibility of Cerenkov radiation by hard X-rays for near bandgap emissions (NBEs), which is due to the photo-electrons generated from the absorption or scattering energetic X-rays and the large refractive index for NBEs near the refractive index resonances. The refractive index of $\mathrm{ZnO}$ and similarly $\mathrm{BaF}_{2}, \mathrm{GaN}$ is given by the Sellmeier empirical equation [13,14],

$$
n^{2}(\lambda)=A+\frac{B_{1} \lambda^{2}}{\lambda^{2}-C_{1}}+\frac{B_{2} \lambda^{2}}{\lambda^{2}-C_{2}}+\frac{B_{3} \lambda^{2}}{\lambda^{2}-C_{3}}
$$

with the empirical parameters $\mathrm{A}, \mathrm{B}_{1}, \mathrm{C}_{1}$, etc., given in Table 1.

Table 1. The Sellmeier fitting parameters for several fast scintillator candidates.

\begin{tabular}{|l|l|l|l|c|c|c|c|}
\hline & \multicolumn{1}{|c|}{$\mathrm{A}$} & \multicolumn{1}{c|}{$\mathrm{B}_{1}$} & \multicolumn{1}{c|}{$\mathrm{C}_{1}$} & $\mathrm{~B}_{2}$ & $\mathrm{C}_{2}$ & $\mathrm{~B}_{3}$ & $\mathrm{C}_{3}$ \\
\hline & & & \multicolumn{1}{c|}{$\left(\mu \mathrm{m}^{2}\right)$} & & $\left(\mu \mathrm{m}^{2}\right)$ & & $\left(\mu \mathrm{m}^{2}\right)$ \\
\hline $\mathrm{BaF}_{2}^{\ddagger}$ & 1.33973 & 0.81070 & $0.10065^{2}$ & $0.19652^{2}$ & $29.87^{2}$ & 4.52469 & $53.82^{2}$ \\
\hline $\mathrm{ZnO}[15]$ & 2.84 & 0.84 & 0.101 & & & & \\
\hline $\mathrm{GaN}$ & 3.60 & 1.75 & $0.256^{2}$ & $17.86^{2}$ & & & \\
\hline
\end{tabular}

The first UV resonance happens in $\mathrm{ZnO}$ and others at the wavelength $\lambda_{1}$,

$$
\lambda_{1}^{2}=C_{1}
$$

which, based on values in Table 1, are listed in Table 2.

Table 2. The first resonances of several fast scintillators according to Table 1.

\begin{tabular}{|c|c|}
\hline & First resonance $(\mathrm{nm})$ \\
\hline $\mathrm{BaF}_{2}$ & 100.65 \\
\hline $\mathrm{ZnO}$ & 318 \\
\hline $\mathrm{GaN}$ & 256 \\
\hline
\end{tabular}

Cerenkov radiation for photoelectrons happens at the critical refractive index $n_{c}=1 / \beta$, with $\beta$ being the photoelectron velocity in the unit of the speed of light in vacuum. At $10 \mathrm{keV}$, the critical refractive index $\mathrm{n}_{\mathrm{c}}=5.13$. At $20 \mathrm{keV} \mathrm{n}_{\mathrm{c}}=3.68$. At $30 \mathrm{keV}, \mathrm{n}_{\mathrm{c}}=3.04$. Such large refractive indices are typically difficult to obtain except at near resonances.

\subsection{Variation of scintillator light yield with samples}

We found that the light output from $\mathrm{ZnO}$ vary significantly from different vendors. It is therefore necessary to characterize the light output when selecting the sensor materials. There are a number of ways to characterize the absorption and light output of scintillators for X rays, namely tagged/gated source measurement [1], reflection and transmission measurement (R\&T), spectroscopic ellipsometry, cathodoluminescence (CL) and photoluminescence (PL) [16]. In R\&T measurement, the absorption

\footnotetext{
$\$$ http://refractiveindex.info/?shelf=main\&book=BaF2\&page=Li
} 
coefficient is given by $\alpha(\lambda)=\frac{1}{d} \ln \left(\frac{(1-R)^{2}}{T}\right)$, where $\mathrm{d}$ is the sample thickness, $\mathrm{T}$ the transmittance, and $\mathrm{R}$ the reflectance.

The measured light yield from several $\mathrm{ZnO}$ :Ga samples was much lower than expected in our previous report [1]. Two new $\mathrm{ZnO}: \mathrm{Ga}$ samples (at $3 \mathrm{~mm}$ and $300 \mathrm{~mm}$ thick respectively) were obtained by Caltech lately. The new $\mathrm{ZnO}$ :Ga samples, shown in Figure 3, has a greenish color, which is distinctive from the previous samples and indicative of rich oxygen defects in the samples. When using a $290 \mathrm{~nm}$ UV source for photoluminescence measurement, the photoluminescence was peaked at 370 to $390 \mathrm{~nm}$, indicating that the light comes from near bandgap transitions. We compared the $59.5 \mathrm{keV} \mathrm{X}$-ray (using an Am-241 radioactive source) induced light yield using a compact ultrahigh efficient PMT (Hamamatsu R9880U-210). The PMT QE efficiency for blue light is the highest. Significant light yield (much greater than single photon yield) is confirmed for the new samples, Samples \#3 and \#4 in Figure 3. The decay time is a few nanosecond, limited by the overall response time of the circuit rather than by the decay time of the scintillators.

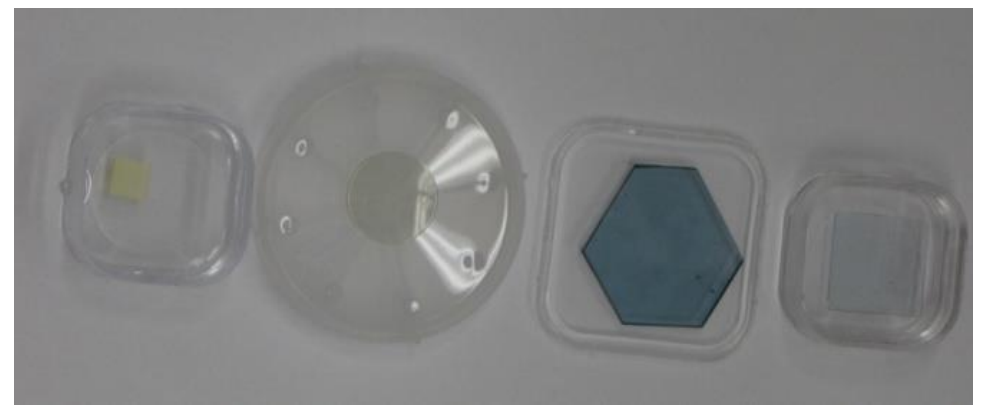

Figure 3. Four $\mathrm{ZnO}$ samples tested using an alpha-tagged 241Am X-ray source. Strong light output at room temperature observed for the two recent samples (first and second from right) provided by Caltech.

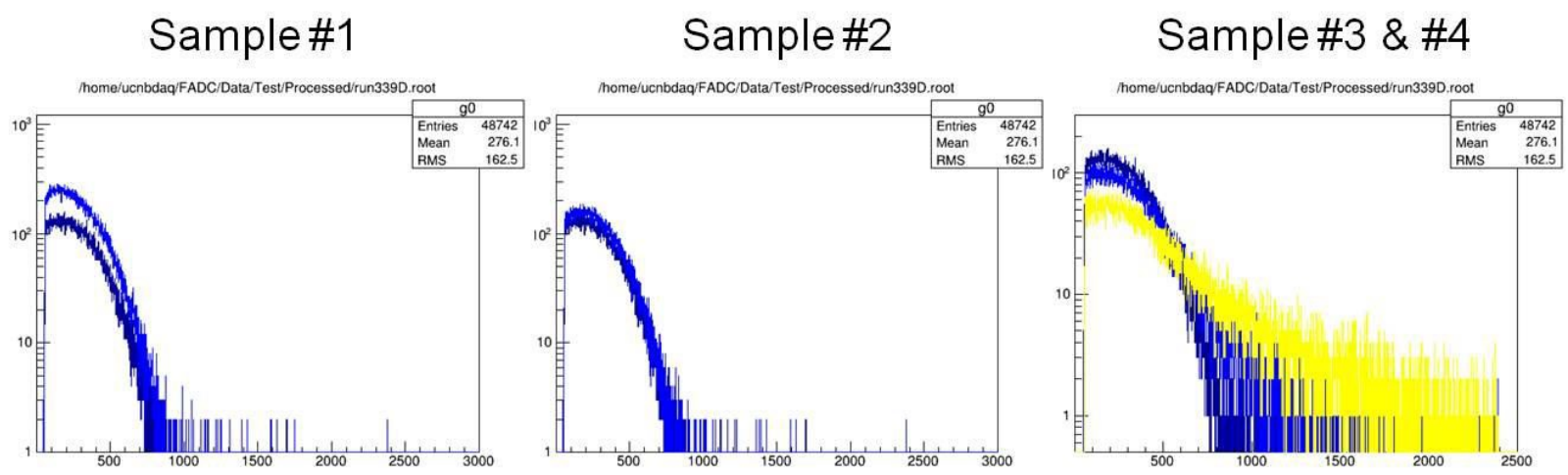

Figure 4. X-ray induced pulse height spectra (PSH) obtained for four different Samples. Each PSH is compared with a background spectrum taken without the source. Sample \#3 and \#4 have pulses that are larger in amplitude than most background signals.

Thinner samples allow more light output than the thicker sample, as indicated by the pulse height increase in Figure 4. Further characterization of new samples, and in particular try to obtain even thinner 
samples less than $100 \mu \mathrm{m}$ thick is planned to understand how different doping, crystal structure, and thickness affect light output.

\subsection{The need for single photon (UV/visible) sensitivity and gigahertz count rate}

The fast light yield from $\mathrm{BaF}_{2}$ is 1.8 photons/ $\mathrm{keV} \mathrm{e}^{-}$. The fast light yield ranging from about 0.4 photons/ $\mathrm{keV} \mathrm{e}^{-}$to a few photons/ $\mathrm{keV} \mathrm{e}^{-}$for $\mathrm{ZnO}$ :Ga from measurements of different material origins. The total number of fast UV/visible photons emitted is about 10 for $10-\mathrm{keV}$ photoelectrons. The low light yield, combined with total internal reflection and other losses, requires that the photodetectors have single photon sensitivity. The energy resolution will be poor because of the paucity of light per $\mathrm{X}$-ray photon. Furthermore, for gigahertz imaging, the photodetectors also need to respond at a gigahertz count rate. Photomultiplier tubes (PMTs), microchannel plates (MCPs), and more recently avalanche photodiodes (APDs), and silicon photomultipliers (SiPMs) are all sensitive to single photons. However, the dead-time due to the high gains can be much longer than a few nanoseconds, making gigahertz rate difficult to achieve. On the other hand, if the gigahertz rate is only needed for a few cycles, it is can be less challenging than achieving $\mathrm{GHz}$ rate continuously. The need for larger communication bandwidth, quantum communications, interplanetary communications, LiDAR imaging, have given rise to a variety of high-speed photon counting technologies that can potentially meet the single-photon counting requirement at gigahertz rates continuously [17].

Achieving high gain or electron multiplication $\geq 10^{4}$ is critical to single photon detection. The first step in the signal generation and amplification chain is always photoelectric absorption that produces one photoelectron per photon. Light and electron scattering reduce the quantum efficiency of photoelectron production to less than one. In APDs and SiPMs, the photoelectron multiplies over an electric field gradient ( $>5 \times 10^{5} \mathrm{~V} / \mathrm{cm}$ in silicon) within the bulk semiconductor. In PMTs and MCPs, the photoelectron multiplies after traversing a vacuum region with an electric field gradient $\sim 10^{2}$ V/cm, determined by the dynode spacing and secondary electron yield from the dynode. Each dynode stage delivers a gain in the range of $g=10$ to $10^{3}, N$ stages increase the gain to $g^{N}$. The fastest PMTs ( $~ 30$ ps rise time, $\sim 100$ ps FWHM pulse) use one or two MCPs as the dynodes. Fast single-photon detectors can be divided into two categories, depending on the material structures for photon absorption and electron multiplication. The PMT/MCP type uses separated photocathode and dynode structures. The APD/SiPM type combines the photon absorption with charge amplification into the bulk of a semiconductor. We discuss the use of the photocathode and dynode structure here, because this approach has demonstrated the fastest time response and low dark count rate. The challenges for imaging applications include efficiency, spatial resolution and dead-time in-between two or more consecutive events. To maximize the efficiency, we can reduce the light loss using the configuration described in Figure 1. We use the photocathode with the highest quantum efficiency to match the energy of the photon. To achieve fine spatial resolution, we may use magnetic field and electron focusing structures. To reduce the dead time, we can minimize the gain requirement or equivalently reduce the electronic noise floor in the storage capacitor (no real time data transmission is planned for multipleframe imaging. The data (charge) is stored temporarily in capacitors and read out at a rate less than 1 $\mathrm{GHz}$ ). The storage capacitor array can be combined with a thin-film transistor (TFT) array for some additional gain if needed [18]. 


\section{PHOTOCATHODE AND ELECTRON MULTIPLICATION}

High quantum efficiency photocathode $\left(\mathrm{Q}_{\mathrm{E}}\right)$ in the UV and blue spectral range will be needed. The $\mathrm{Q}_{\mathrm{E}}$ is described by W. E. Spicer's three-step model: photoelectric absorption, photoelectron diffusion, and photoelectron escape. For $N_{p}$ photons, the average number of photoelectrons emitted is $\left\langle\mathrm{N}_{\mathrm{e}}\right\rangle=\mathrm{Q}_{\mathrm{E}} \mathrm{N}_{\mathrm{p}}$. The Poisson statistics implies that the null event, $\mathrm{N}_{\mathrm{e}}=0$, has a probability of $\left.\mathrm{p}\left(\mathrm{N}_{\mathrm{e}}=0\right)=\exp (-<\mathrm{Ne}\rangle\right)$. For $\mathrm{N}_{\mathrm{p}}=10$ and $\mathrm{Q}_{\mathrm{E}} \leq 0.1, \mathrm{p}\left(\mathrm{N}_{\mathrm{e}}=0\right) \geq \mathrm{e}^{-1}=36.8 \%$. This is quite a significant loss of efficiency. For $\mathrm{Q}_{\mathrm{E}}$ $=0.2,0.3,0.4, \mathrm{p}\left(\mathrm{N}_{\mathrm{e}}=0\right)=13.5 \%, 5.0 \%, 1.8 \%$ respectively. Therefore, $\mathrm{Q}_{\mathrm{E}} \geq 0.3$ is desirable.

No single photocathode material delivers high QE for $200 \mathrm{~nm}$ to $400 \mathrm{~nm}$, the spectral range expected for $\mathrm{BaF}_{2}, \mathrm{ZnO}: \mathrm{x}$ and other fast scintillators. Different photocathodes will have to be used to match the fast emission spectrum from different scintillators. A possible list of photocathodes is given in Table 3. A benefit of tailoring photocathode to fast emission is that the sensitivity to slow emission can be suppressed due to the small $\mathrm{Q}_{\mathrm{E}}$ of the photocathode to longer wavelengths.

Table 3. Possible high QE photocathode for different spectral ranges and fast scintillators.

\begin{tabular}{|c|c|c|c|}
\hline Photocathode & Spectral range $(\mathrm{nm})$ & $\mathrm{Q}_{\mathrm{E}}{ }^{\S}$ & Scintillator \\
\hline Ultra Bialkali (UBA) & $310-400$ & $>0.4$ & $\mathrm{ZnO}: \mathrm{Ga}$ \\
\hline Super Bialkali (SBA) & $290-440$ & $>0.3$ & $\mathrm{ZnO}: \mathrm{Ga}$ \\
\hline $\mathrm{GaN}$ & $260-350$ & $>0.2$ & $\mathrm{GaN}: \mathrm{x}$ \\
\hline $\mathrm{CsTe}$ & $200-300$ & $>0.1$ & $\mathrm{BaF}_{2}$ \\
\hline
\end{tabular}

To examine the overall performance of the scintillator coated by a photocathode, a possible setup is shown in Figure 5 using a multiple-pulse UV laser that can excite the fast emissions in scintillators through photoluminescence. This initial setup can be followed by a similar setup using synchrotron $\mathrm{X}$-rays and laser-produced $\mathrm{X}$-rays for multi-pulse measurements.

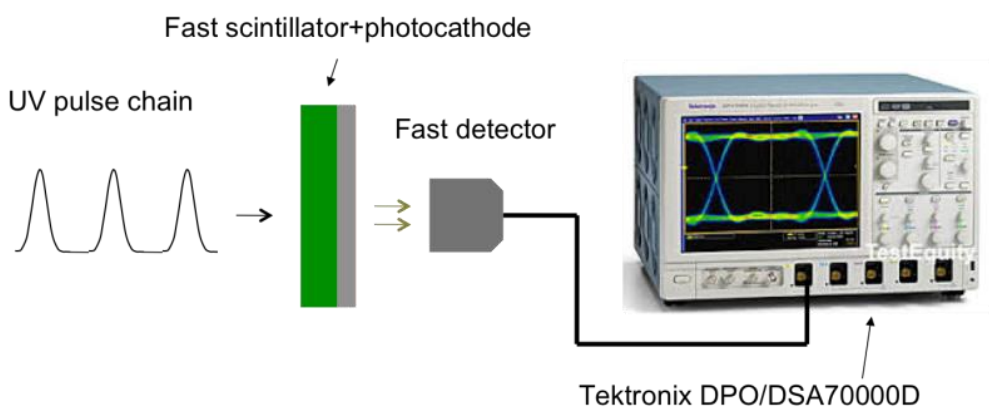

Figure 5: A multiple pulse chain from a UV laser can be used to excite the fast emissions of the scintillators and examine the overall time response and efficiency. The signal outputs from a fast MCP-PMT detector can be digitized using a Tektronix DPO/DSA70000D oscilloscope.

Photoelectron multiplication can use a MCP structure (transmission mode) [19]. The data storage and readout can use a low-noise CCD or CMOS with on board memories. We shall mention that the 'Tipsy'

$\S$ The data are based on Hamamastu photocathodes. 
concept can also be applied to the proposed imaging concept [20]. Tipsy uses a stacked set of dynodes for charge amplification and CMOS pixels for data storage.

\section{THE MULTILAYER DETECTOR STRUCTURE}

A multilayer detector using thin scintillators is shown in Figure 6. High efficiencies for hard X-rays are achieved by using stacked multiple scintillator layers. Each X-ray photons have multiple chances to be absorbed or scattered. The electron multiplication and charge storage are out of the line-of-sight for the diffracted X-ray beam. A magnetic field can be used to guide the photoelectrons sideways (perpendicular to the X-ray beam direction) to amplification and storage.

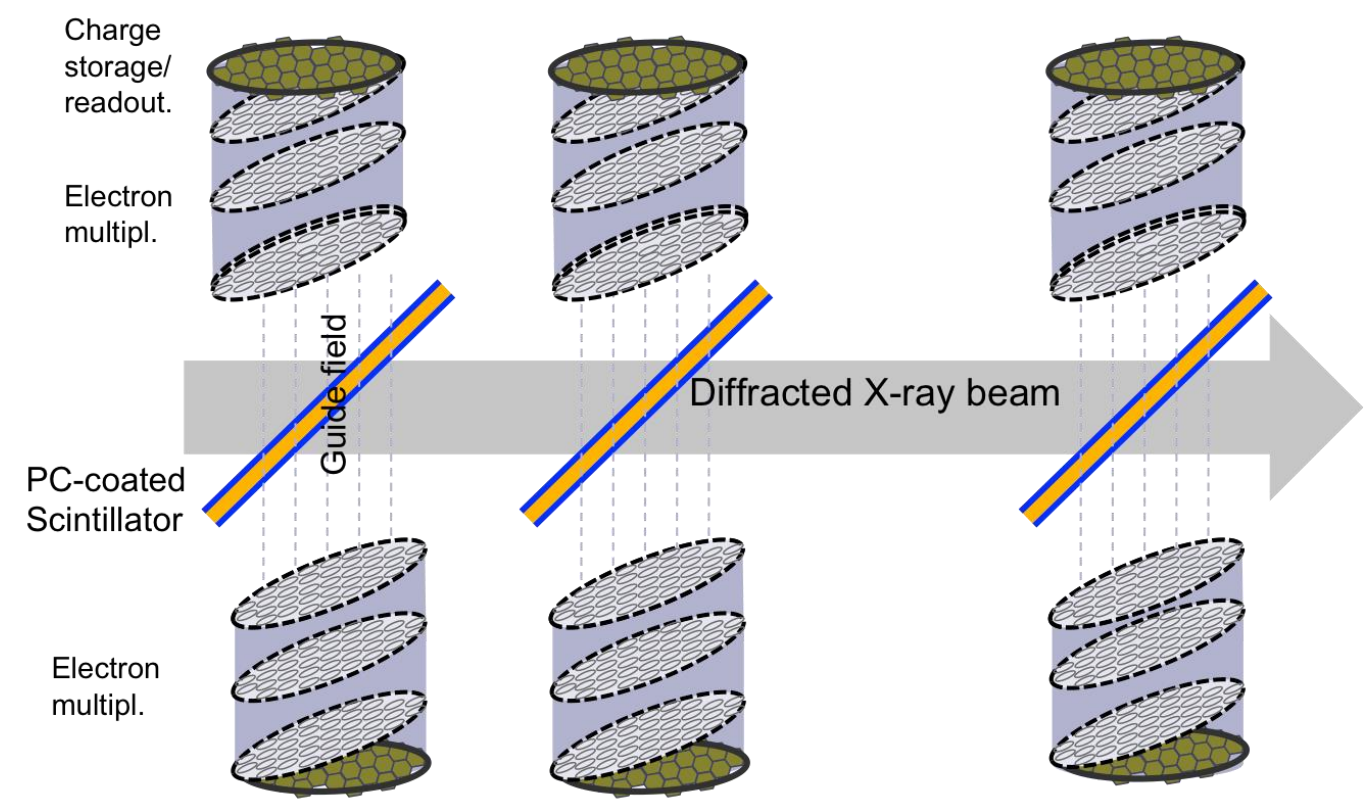

Figure 6. A multi-layer detector architecture for efficient and fast imaging of diffracted X rays. A guide magnetic field perpendicular to the X-ray direction guide the photoelectrons to amplification and storage. The magnetic field also preserves the image contrast due to X-ray absorption at the scintillator location.

Since the initial kinetic energies of the photoelectrons are only a few eV, the Larmor radii of the electrons are $53 \mu \mathrm{m}$ for a $5 \mathrm{eV}$ electron (perpendicular to the field) in a $1 \mathrm{kG}$ field. Doubling the field will decrease the Larmor radius or increase the spatial resolution by half. A gated electric pulse between $100 \mathrm{~V}$ to several $\mathrm{kV}$ may be applied between the photocathode and the entrance of the electron multiplication stages. Such a gated voltage bias will increase the time response of the system as well as provide the initial kinetic energies for the electron multiplication. Since the electric bias is along the direction of the magnetic field, the spatial resolution does not degrade as the kinetic energy of the photoelectrons increases. The number of layers for $\mathrm{ZnO}$ and alike is about 10, if each layer is $100 \mu \mathrm{m}$ thick. The number of layer increases inversely to the layer thickness for a fixed total layer thickness.

In comparison with columnar structures, a multi-layer scintillator structure is easier to fabricate and many thin film techniques are available for different scintillator materials. One challenge is to 
demonstrate the high $\mathrm{Q}_{\mathrm{E}}$ photocathode coating on fast scintillator films. The material selection process and electron multiplication are discussed in Sec. 3.

\section{SUMMARY AND CONCLUSION}

A gigahertz hard X-ray camera system can be broken down into three subsystems: hard X-ray sensor array, signal (electron) amplification, and data (charge) storage. By coating fast scintillator thin films with high quantum efficiency $(>0.3$ ) photocathodes, the UV/visible photon loss can be reduced significantly and single hard X-ray photon detection is possible using fast scintillators as sensors, which have a low light yield on the order of $1 \mathrm{UV} /$ visible photon/ $\mathrm{keV} \mathrm{e}$. Electron multiplication at least $10^{4}$ would be required to detect the photoelectrons induced by the UV/visible photons. MCPs and new nanostructured electron multiplication technologies such as Tipsy are possible schemes to achieve such a gain without sacrificing the fine spatial $(<100 \mu \mathrm{m}$, detector location) resolution and sub-ns temporal resolution simultaneously. On-board charge storages can be used to store multiple frames of images at gigahertz imaging rates. The growth of the multi-layer thin-films (photocathode combined with scintillators) can be combined with MCP-PMT detectors and GHz electronics (oscilloscope) for thin-film multilayer sensor development.

\section{REFERENCES}

[1] Z. Wang, E. Guardincerri, D. D. Rathman, M. E. Azzouz, Cris W. Barnes, R. Berger, E. M. Bond, D. M. Craig, David Holtkamp, J. S. Kapustinsky, Alexei V. Klimenko, K. Kwiatkowski, R. B. Merl, C. L. Morris, J. O. Perry, E. Ramberg, R. K. Reich, A. Ronzhin, K. Warner, R. T. Williams, and Ren-Yuan Zhu, 'Gigahertz (GHz) hard X-ray imaging using fast scintillators,' Proc. SPIE 8852 (2013) 88521A.

[2] A. Koch, C. Raven, P. Spanne and A. Snigirev, 'X-ray imaging with submicrometer resolution employing transparent luminescent screens.' J. Opt. Soc. Am. 15, (1998) 1940-1951.

[3] J. A. Shepherd, S. E. Sobottka and M. B. Williams, 'Thin film NaI(Tl) scintillators for high-resolution large-area imaging of soft X-rays.' Proc SPIE 2009 (1993) 109.

[4] Y. Zorenko, V. Gorbenko, T. Voznyak, T. Martin, P.-A. Douissard, J. A. Mares and M. Nikl, 'LuAG:Pr, LuAG:La and LuAP:Ce thin film scintillators for visualization of X-ray images.' Proc. SPIE 7310 (2009) 731007-1.

[5] M. Dapor, Transport of Energetic Electrons in Solids: Computer Simulation with Applications to Materials Analysis and Characterization. (Springer, 2014).

[6] S. E. Derenzo, M. J. Weber, and M. K. Klintenberg, Nucl. Instrum. Methods Phys. Res. A 486, (2002) 214 ; M. Lorenz et al, 'Fast, high-efficiency, and homogeneous cathodoluminescence of ZnO scintillator thin films on Sapphire.' Appl. Phys. Lett. 89 (2006) 243510.

[7] J. Wilkinson, K. B. Ucer, and R. T. Williams, Nucl. Instrum. Methods Phys. Res. A 537, (2005) 66.

[8] P. J. Simpson, R. Tjossem, A. W. Hunt, K. G. Lynn, and V. Munné, Nucl. Instrum. Methods Phys. Res. A 505 (2003) 82.

[9] A. P. Roth, J. B. Webb and D. F. Williams, 'Absorption edge shift in ZnO Thin Films at high -carrier densities.' Sold. State. Comm. 39 (1981) 1269-1271.

[10] Sernelius, K.-F. Berggren, Z.-C. Jin, I. Hamberg and C. G. Granqvist, 'Bandgap tailoring of ZnO by means of heavy Al doping.' Phys. Rev. B 37 (1988) 10244.

[11] N. R. Aghamalyan, E. A. Kafadaryan, R. K. Hovsepyan, and S. I. Petrosyan, 'Absorption and reflection analysis of transparent conductive Ga-doped ZnO Films.' Semicond. Sci. Technol. 20 (2005) 80-85.

[12] V. Srikant and D. R. Clarke, 'Optical absorption edge of ZnO thin films: the effect of substrate.' J. Appl. Phys. 81 (1997) 6357 $-6364$.

[13] H. Haug and S. W. Koch, 'Quantum Theory of the Optical and Electronic Properties of Semiconductors.'

[14] J. Faist, 'Quantum Cascade Lasers,' (2013).

[15] H. Yoshikawa and S. Adachi, 'Optical Constants of ZnO.’ Jpn. J. Appl. Phys. 36 (1997) 6237-6243.

[16] V. Srikant and D. R. Clarke, 'On the Optical Band Gap of ZnO.' J. Appl. Phys. 83 (1998) $5447-5451$.

[17] W. H. Farr, J. Gin and D. Nguyen, 'Gigahertz bandwidth photon counting.' Proc. SPIE 7320 (2009) 732006. 
[18] Y. Sun, E. Menard, J. A. Rogers, et al., 'Gigahertz operation in flexible transistors on plastic susbtrates.' Appl. Phys. Lett. 88 (2006) 183509.

[19] A. Tremsin, J. V. Vallerga, J. B. McPhate and O. H. W. Siegmund, 'Optimization of high count rate event counting detector with microchannel plates and quad Timepix readout.' Nucl. Instrum. Methods Phys. Res. A (2014) (in Press).

[20] H. Van der Graaf, M. A. Bakker, H. W. Chan, E. Charbon, F. Santagata, P. M. Sarro and D. R. Schaart, 'The Tipsy single soft photon detector and the Trixy ultrafast tracking detector.' J. Instrum. 8, C01036. 